Research Article

\title{
Preparation and Drug Release Study of Novel Nanopharmaceuticals with Polysorbate 80 Surface Adsorption
}

\author{
Xiaojun Tao, ${ }^{1}$ Yu Li, ${ }^{1}$ Qian Hu, ${ }^{1}$ Li Zhu, ${ }^{1}$ Zixuan Huang, ${ }^{1}$ Jiajin Yi, ${ }^{1}$ Xiaoping Yang $\mathbb{D}^{1}$ \\ Jianzhuo $\mathrm{Hu}^{2}$, and Xing Feng $\mathbb{D}^{1}$ \\ ${ }^{1}$ Key Laboratory of Study and Discovery of Small Targeted Molecules of Hunan Province, School of Medicine, Hunan Normal \\ University, Changsha 410013, China \\ ${ }^{2}$ The First Hospital of University of Chinese Medicine, Changsha 410007, China
}

Correspondence should be addressed to Xing Feng; fengxing01@hotmail.com

Received 13 December 2017; Revised 16 April 2018; Accepted 24 April 2018; Published 20 May 2018

Academic Editor: Mauro Pollini

Copyright ( 2018 Xiaojun Tao et al. This is an open access article distributed under the Creative Commons Attribution License, which permits unrestricted use, distribution, and reproduction in any medium, provided the original work is properly cited.

\begin{abstract}
Most free drugs that cross the blood-brain barrier are characterized by high liposolubility, but they often have limited clinical applications because of poor dissolution and poor bioavailability. In this study, we prepared donepezil drug-loaded nanoparticles (DZP) with cholesterol-modified pullulan (CHP) as the nanocarrier (DZP-CHP) and surface modified the drugloaded nanoparticles to improve the water solubility of donepezil while enhancing its targeting and sustained release. We determined the drug loading and encapsulation efficiency of DZP-CHP nanoparticles at different feed ratios. The mean \pm SD drug loading and entrapment efficiency were high: $13.52 \pm 2.03$ and $86.54 \pm 1.31$. On dynamic light-scattering measurement, mean \pm SD particle size was $260.7 \pm 1.76 \mathrm{~nm}$, polydispersity index $0.123 \pm 0.004$, and zeta potential $-5.75 \pm 0.64 \mathrm{mV}$. DZP-CHP nanoparticles prepared with the optimal feed ratio $(\mathrm{DZP}: \mathrm{CHP}=1: 5)$ were coated with polysorbate 80 , and the adsorption process was determined by isothermal titration calorimetry. We found good affinity between polysorbate 80 and DZP-CHP, with mean \pm SD coverage $2.7 \pm 0.372$. The mean \pm SD drug loading and entrapment efficiency of polysorbate 80 -emulsified DZP-CHP nanoparticles were $8.25 \pm 1.80$ and $91.28 \pm 4.57$, respectively, and the proportion of drug released by $72 \mathrm{~h}$ was $42.71 \%$. Compared to DZP-CHP alone, PS-DZP-CHP can enhance the release of donepezil.
\end{abstract}

\section{Introduction}

Research hot topics in the drug field are to design drug preparations with high-efficiency delivery [1-3]. Oral solid drugs released from the formulation and dissolved in body fluid is the premise of absorption, so with poor dissolution, drug absorption in vivo is slow and with low bioavailability, therefore not reaching the therapeutic level of plasma concentration and poor clinical treatment $[4,5]$. Fat-soluble drugs more easily cross the blood-brain barrier for a pharmacological effect [6]. In preparing conventional formulations for craniocerebral diseases, increasing the drug solubility often requires a number of drug additions, which results in toxicity. Currently, about $40 \%$ of clinically used drugs have limited use because of poor dissolution $[7,8]$.

In recent years, the emergence of nanoformulations for injection has brought hope to solving the problem of drug delivery of fat-soluble drugs. Nanoparticles (NPs) represent a micropectin system composed of nanospheres or nanocapsules with particle size typically $<1 \mu \mathrm{m}$ [9] Because of the small particle size and large specific surface area of NPs, the solubility and dissolution rate of insoluble drugs can be increased after drug loading [10]. Also, with the preparation and surface modification of biomaterials with specific properties, the NPs can feature long circulation and controlled release $[11,12]$. Targeted delivery and reduced dose ensure efficacy and reduce side effects.

In this study, we used donepezil, a clinically important drug for treating Alzheimer's disease, with poor solubility [13] to develop a new type of nanostructured drug. Donepezil is fat-soluble, poorly dissolved in the body, and generally taken orally; with poor drug absorption and low bioavailability, the drug has no tissue-specific, toxic side effects on the peripheral nervous system, and its clinical application is 


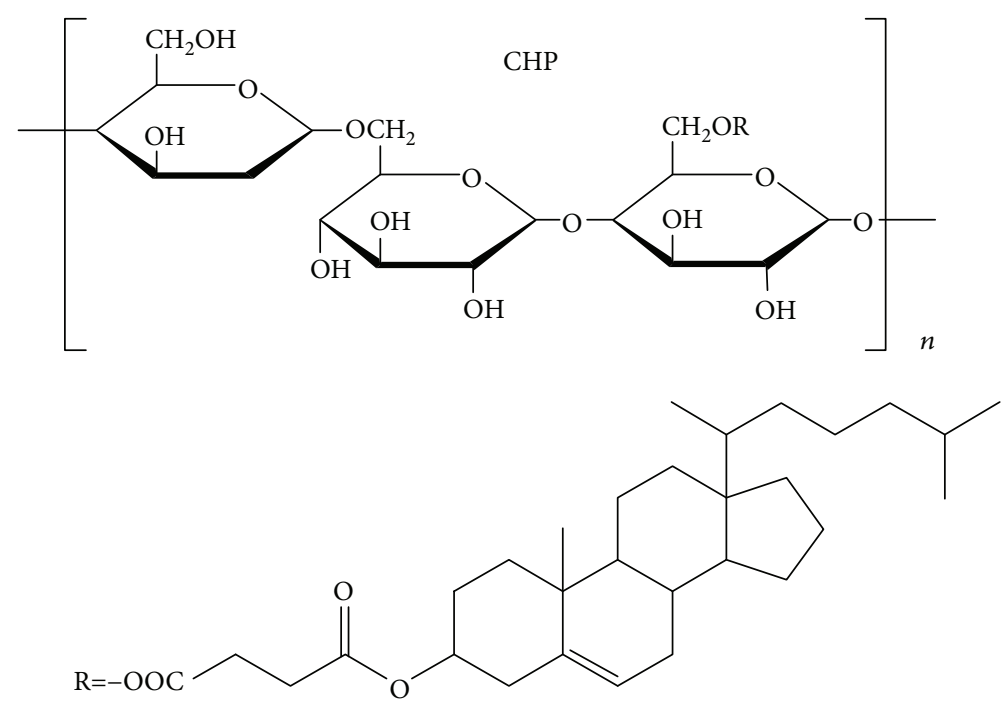

Figure 1: The chemical structure of CHP.

limited. In addition, conventional Aricept should be taken daily to maintain the therapeutic effect. However, in Alzheimer's patients, with the progression of the disease to a certain stage, the lack of memory of daily medication on a daily basis is an obstacle to the use of donepezil. We need new types of preparations to overcome these deficiencies.

Pullulan, an exopolysaccharide produced by budding short sporophore enzymes, is nontoxic and biodegradable $[14,15]$. Cholesterol hydrophobically modified pullulan (CHP) is an amphipathic substance that self-assembles in aqueous solution into nanostructures with a shell of cholesteric hydrophobe and sugar-chain hydrophilicity [16] (Figure 1). CHP NPs can form a complex with $\mathrm{A} \beta$ protein, effectively preventing protein aggregation, and can be quickly cleared from cells, which strongly inhibit cytotoxicity and provide a basis for the selection of nanomaterials [17]. Donepezil, as a strong hydrophobic drug, can be loaded into the hydrophobic center of CHP NPs to form a donepezil nanopreparation (DZP-CHP). Although nanopharmaceutical formulations have some passive targeting due to their small-size properties, the surface must be modified to achieve precise, tissue-specific targeting.

Polysorbate 80 is a common nonionic surfactant. The fatty acid chain (hydrophobic) and ethylene oxide units (hydrophilic) provide its amphiphilic properties. Polysorbate 80 has features of hydrophilicity, nonionicity, biodegradability, and nontoxicity to cells at low concentration and is easy to obtain. Therefore, it is widely used for the distribution of substances in food and drug products [18-20]. Numerous studies have found that it can be applied to the NP surface and can cross the blood-brain barrier through receptormediated endocytosis or other mechanisms and enhance drug-targeted delivery to the central brain for an enhanced targeted therapeutic effect [21-23]. In addition, the hydrophilic and hydrophobic portions of the nonionic surfactant from the polysorbate family are involved in the interaction, and their coating directly inhibits the reticuloendothelial system and thus helps to prolong the circulation time of
NPs in the body $[24,25]$. Polysorbate 80 also has a sustained-release effect [26].

Here we investigated the use of CHP NPs loaded with donepezil, then added polysorbate 80 , and used isothermal titration calorimetry (ITC) to confirm that polysorbate 80 can be successfully adsorbed on the CHP NP surface and achieve the desired rate of adsorption. We aimed to provide the experimental basis for the research of new dosage forms of drugs for Alzheimer's disease.

\section{Materials and Methods}

2.1. Materials. The materials used are the following: constant temperature magnetic stirrer (IKA RCT basic, Germany), vacuum freeze dryer (Maxi Dry Lyo, Heto-Holten), transmission electron microscope (TEM) (JIEM-100S Japan), nanoparticle size and zeta potential analyzer (Malvern ZS-90, Britain); UV-vis spectrophotometer (JASCO V-560, USA); isothermal titration calorimeter (VP-ITC, Microcal Inc., Northampton, MA), dialysis bags (molecular weight cutoff 8 to $12 \mathrm{KDa}$, Germany), cholesterol hydrophobic modified pullulan (homemade), donepezil (Donepezil, Shanghai Ziqi Biological Technology Co. Ltd.), polysorbate 80 (Polysorbate-80, Tianjin Fuchen Reagent Institute). The remainder of the reagents was domestic analytical reagent.

\subsection{Methods}

2.2.1. Dialysis Preparation of CHP and DZP-CHP NPs. As we previously described $[27,28]$, dialysis was used to prepare NPs. We used $20 \mathrm{mg}$ graft polymer CHP polymer dissolved in $2 \mathrm{ml}$ DMSO solvent. The solution was transferred to a dialysis bag (MWCO $8-12 \mathrm{kDa}$ ) and placed in 11 distilled water. The water was changed every $3 \mathrm{~h}$ in the first $12 \mathrm{~h}$ of dialysis and every $6 \mathrm{~h}$ in the $12 \mathrm{~h}$ after dialysis for a total of $24 \mathrm{~h}$.

$4 \mathrm{mg}$ DZP and $20 \mathrm{mg}$ CHP were dissolved in an appropriate amount of DMSO and triethylamine (TEA/DZP =2, $\mathrm{mmol} / \mathrm{mmol}$ ) was added; the drug and the material solution 
were thoroughly mixed in a ratio of $1: 5$, using the above method to prepare drug-loaded NPs. When the DMSO dialysis was clean, the solution was removed, then was treated with an ultrasound probe $(50 \mathrm{~W})$ for $2 \mathrm{~min}$, and volume fixed in a $10 \mathrm{ml}$ volumetric flask, then filtered with a $0.45 \mu \mathrm{m}$ filter to obtain CHP and DZP-CHP NPs, which were stored at $4^{\circ} \mathrm{C}$.

2.2.2. Preparation of the Standard Curve of the Donepezil. $5 \mathrm{mg}$ reference substance of donepezil was accurately weighed and added to the solution, dissolved with DMSO and diluted to $1 \mu \mathrm{g} / \mathrm{ml}$. The maximal ultraviolet absorption wavelength was detected by scanning in the 200 to $400 \mathrm{~nm}$ wavelength range with DMSO as a blank control.

An amount of $1 \mathrm{mg}$ donepezil was added to a $10 \mathrm{ml}$ volumetric flask, dissolved, and fixed to scale with DMSO, to obtain $100 \mu \mathrm{g} / \mathrm{ml}$ standard stored solution. An appropriate amount was diluted with DMSO to $5,10,15,20$, and $25 \mu \mathrm{g}$. With the DMSO solution as the control, the absorbance was measured at the maximum absorption wavelength, with absorbance $A$ as the ordinate and concentration $C$ as the abscissa to obtain a regression equation.

2.2.3. Isothermal Titration Calorimetry (ITC). A concentration of polysorbate 80 solution was dropped into the DZPCHP NP solution, and the isothermal droplet calorimeter was used to measure its thermal change. An amount of $80 \mathrm{ml}$ polysorbate was injected into the NP titration pool containing DZP-CHP for titration 20 times, $4 \mathrm{ml}$ each. The temperature of the droplet was set to $25^{\circ} \mathrm{C}$, and the thermodynamic parameters and connection curves were obtained by 20 times titration.

2.2.4. Polysorbate 80 Emulsification of DZP-CHP NPs. A concentration of DZP-CHP was placed in a $10 \mathrm{ml}$ beaker and aspirated into a polysorbate 80 (PS) emulsifier beaker containing $0.7 \mathrm{mmol}$ for $1 \mathrm{~h}$. The mixed solution was placed in the EP tube, then treated for 3 min with the ultrasound probe (output power: 100, intermittent pulse working mode: pulse width $2.0 \mathrm{~s}$, intermittent time $2.0 \mathrm{~s}$ ), with repeated operation three times until uniform dispersion, then filtered with a filtration membrane to remove impurities and obtain polysorbate 80-emulsified DZP-CHP NPs (PS-DZP-CHP).

2.2.5. Measurement of NP Size, Zeta Potential, and Morphology Observation. The size, polydispersity index (PDI), and zeta potential of CHP, DZP-CHP, and PS-DZP-CHP NPs were measured by use of the Malvern ZS-90 system. The morphology of the particles was evaluated by TEM, and the newly prepared CHP, DZP-CHP, and PS-DZP-CHP water solutions were dropped on a carbon-supporting copper net. After drying, dyeing with the phosphotungstic acid, and natural drying again, the sample was observed by using TECNAI Spirit $(120 \mathrm{kV})$ TEM (FEL, Hong Kong). The freeze-dried NPs were dissolved in pure water, added to clean silicon wafers and dried at room temperature, and the surface structure was observed by scanning electron microscopy.

2.2.6. Determination of the Drug Loading Capacity (LC) and Encapsulation Efficiency (EE) of DZP-CHP and PS-DZP$C H P$. The determination of the drug loading capacity
(LC) and encapsulation efficiency (EE) of DZP-CHP and PS-DZP-CHP follows Tao et al. [16]. For $4 \mathrm{ml}$ freshly prepared DZP-CHP and PS-DZP-CHP NPs, absorbance was determined at $312 \mathrm{~nm}$ by spectrophotometry. The same solvent with blank CHP was a control. The drug content was calculated according to the standard curve, then the loading and encapsulation rate were obtained as follows:

$$
\begin{array}{r}
\text { Loading capacity }(\mathrm{LC} \%)=\frac{\text { the amount of DZP in the NPs }}{\text { weight of NPs }}, \\
\text { Encapsulation efficiency }(\mathrm{EE} \%)=\frac{\text { the amount of DZP in the NPs }}{\text { total amount of DZP }} .
\end{array}
$$

2.2.7. Release of DZP-CHP and PS-DZP-CHP NPs In Vitro. Using the dialysis bag diffusion technique [29] to measure the release of donepezil, $1 \mathrm{mg}$ DZP-CHP and PS-DZP-CHP NPs were dissolved in $5 \mathrm{ml} 0.01 \mathrm{M}$ phosphate buffer solution (pbs, ph 7.4), then transferred to a dialysis bag (MWCO 8$12 \mathrm{kDa}$ ) and placed in the same PBS solution at $37^{\circ} \mathrm{C}$ with constant temperature and magnetic stirring. An amount of $4 \mathrm{ml}$ solution was removed at $0,0.5,1,2,4,8,12,24,48$, and $72 \mathrm{~h}$. An amount of $4 \mathrm{ml}$ fresh PBS was added with the same $\mathrm{pH}$ at the same time. UV-vis spectrophotometry was used to determine the absorbance of dialysis solution of different times at $312 \mathrm{~nm}$; the content of the solution was determined by the standard curve, and the release test was repeated three times in vitro.

$$
Q \%=\frac{\left(C_{n} \times V+V_{n} \sum_{i=0}^{i=n} C_{i}\right)}{W_{\text {drug loading }}},
$$

where $C_{n}$ is the sample concentration at $T_{n}(\mu \mathrm{g} / \mathrm{ml}), V$ is the release volume of the medium at $T_{i}(\mathrm{ml})$, and $C_{i}$ is the sample concentration at $T_{i}(\mu \mathrm{g} / \mathrm{ml})$.

In addition, we changed the PBS release media to FBS release media at a certain concentration and analyzed the drug release of PS-DZP-CHP NPs. An amount of FBS solution $(0.1 \mathrm{mg} / \mathrm{ml})$ was added to the dialysis bag to measure the drug release of PS-DZP-CHP NPs. A weight ratio of donepezil to FBS of 1:10 was prepared to obtain nanoparticle-drug-FBS complex, and the mixture solution was dialyzed against $1000 \mathrm{ml}$ distilled solution for $6 \mathrm{~h}$ to remove free donepezil. Then, we measured the drug release as described previously.

2.3. Statistical Analysis. Data are presented as mean \pm SD and were analyzed by Student $t$-test or ANOVA for multiple samples with the use of SPSS 12.0. $P<0.5$ was considered statistically significant.

\section{Results}

3.1. Determining the Standard Curve of Donepezil. To determine the drug loading and entrapment efficiency of drugloaded NPs, we established a standard curve for donepezil. UV scan 1 showed maximum absorption of donepezil at wavelengths 270 and $312 \mathrm{~nm}$, and the larger value of $312 \mathrm{~nm}$ was selected as the detection wavelength to minimize 

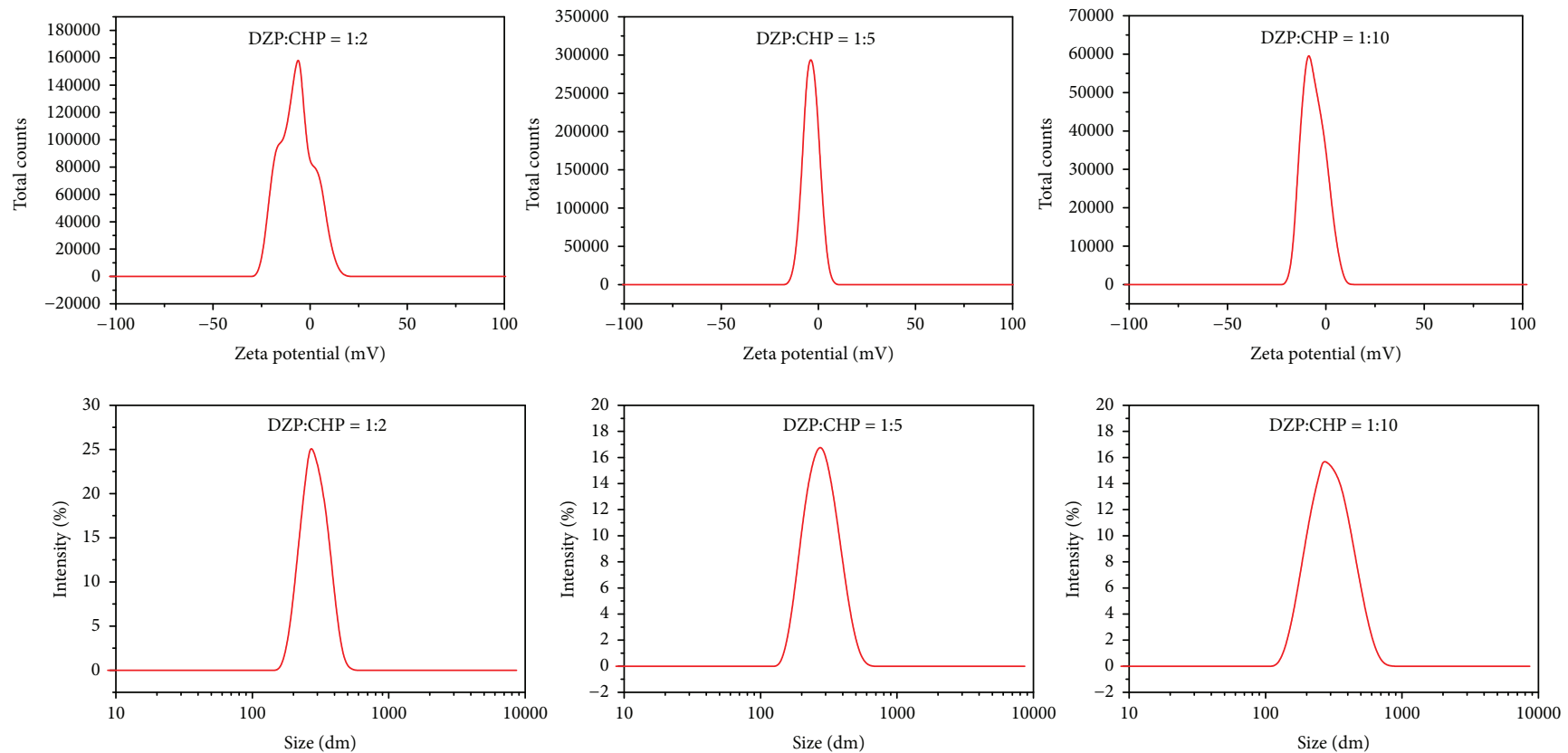

Figure 2: Potential change and particle size distribution of DZP-CHP NPs at different feed ratios.

TABle 1: Particle size, polydispersity index (PDI), zeta potential, drug loading, and entrapment efficiency of CHP NPs at different feed ratios $(1: 2,1: 5$, and $1: 10)$.

\begin{tabular}{lccccc}
\hline Feed ratio & Particle size $(\mathrm{nm})$ & PDI & Zeta potential $(\mathrm{mV})$ & Drug loading (\%) & Encapsulation efficiency (\%) \\
\hline $1: 2$ & $273.3 \pm 3.72$ & $0.138 \pm 0.013$ & $-6.20 \pm 0.40$ & $12.02 \pm 1.90$ & $42.00 \pm 5.65$ \\
$1: 5$ & $260.7 \pm 1.76$ & $0.123 \pm 0.004$ & $-5.75 \pm 0.64$ & $13.42 \pm 2.03$ & $86.54 \pm 1.31$ \\
$1: 10$ & $266.8 \pm 4.56$ & $0.196 \pm 0.019$ & $-9.30 \pm 0.39$ & $7.40 \pm 1.72$ & $59.71 \pm 4.43$ \\
\hline
\end{tabular}

measurement errors. The standard curve was drawn with the concentration of donepezil standard solution as the abscissa and absorbance value as the ordinate, with standard curve equation $Y=-0.00373+0.01574 X$. We found a linear relationship between concentration and absorbance in the concentration range of $0-30 \mu \mathrm{g} / \mathrm{ml}$, with correlation coefficient 0.99885 , which met the requirements.

3.2. Relation between Particle Size, Zeta Potential, Drug Loading, and Entrapment Efficiency of DZP-CHP NPs and Feed Ratio. The prepared CHP NPs were loaded with donepezil according to different feed ratios, and particle size and zeta potential were measured by dynamic light scattering. At feed ratios $1: 2,1: 5$, and $1: 10$, the mean \pm SD NP size was $273.3 \pm 3.72,260.7 \pm 1.76,266.8 \pm 4.56 \mathrm{~nm}$, respectively, and PDI was $0.138 \pm 0.013,0.123 \pm 0.004,0.196 \pm 0.019$, respectively (Figure 2). The distribution of NP size was uniform, and the feed ratio was 1:5 with the smallest PDI and the most uniform distribution. The feed ratio $1: 2$ led to the largest NP size, which may be due to the too small amount of hydrophobic donepezil in the hydrophobic center of NPs and too weak hydrophobic interactions between the hydrophobic ends of the NPs and hydrophobic drugs during the self-assembly process, thereby resulting in the aggregation and a too large particle size. The results of particle size distribution are also consistent with this suggestion, showing the smallest NPs at feed ratio 1:5, which indicates that the formed NPs are more stable and uniform at this feed ratio.

We measured the absorbance of the drug-loaded NPs at $312 \mathrm{~nm}$. The drug concentration was calculated by the standard curve of the concentration of donepezil to the absorbance, and the drug loading and entrapment efficiency of DZP-CHP NPs were calculated as described in Materials and Methods. With feed ratios of $1: 2,1: 5$, and $1: 10$, the mean \pm SD drug loading of drug-loaded NPs was $12.02 \pm 1.90 \%, 13.42 \pm 2.03 \%$, and $7.40 \pm 1.72 \%$, respectively, and the entrapment efficiency was $42.00 \pm 5.65 \%$, $86.54 \pm 1.31 \%$, and $59.71 \pm 4.43 \%$, respectively (Table 1 ). The drug loading and encapsulation efficiency of DZP-CHP NPs peaked at feed ratio $1: 5$. Therefore, this NP-loaded donepezil can provide better drug loading and encapsulation efficiency, with the best feed ratio about $1: 5$. The drug loading and entrapment efficiency of CHP NPs were saturated: when the mass ratio of drug to CHP NP exceeded a certain amount, the larger the feed ratio, the smaller the drug loading and entrapment efficiency of the NPs. Our results show an ideal feed ratio of CHP to donepezil of $1: 5$, so we chose DZP-CHP with a feed ratio of 1:5 for the next step. 


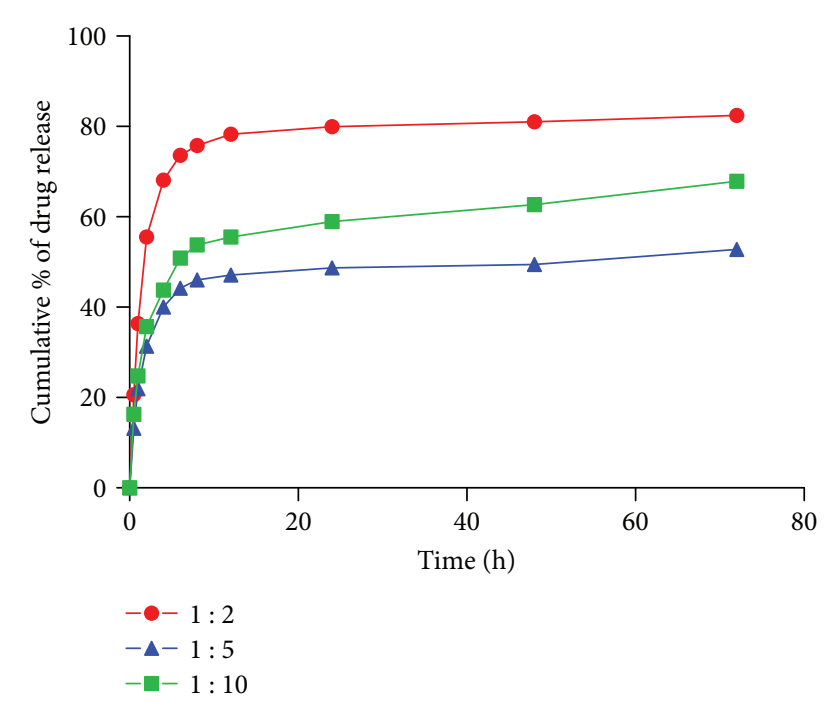

Figure 3: Release curves of donepezil from DZP-CHP NPs in phosphate buffered saline (PBS) at $37^{\circ} \mathrm{C}$ in vitro at different feed ratios. $1: 2,1: 5,1: 10$.

3.3. Relation between Drug Release of DZP-CHP NPs In Vitro and Feed Ratio. The dissolution curve of DZP-CHP NPs in distilled water at different feed ratios is shown in Figure 3. There are two stages in the drug release process of drugloaded NPs: the initial stage with a rapid release (phase I), followed by a sustained long-term slow release (phase II). The possible reasons for this release characteristic are related to the presence of donepezil in DZP-CHP NPs. Some drugs are adsorbed on the surface of DZP-CHP self-aggregated NPs by a weak interaction, whereas most drugs and cholesterol in DZP-CHP molecules enter the hydrophobic core of NPs by hydrophobic interaction. The rapid release of the drug by the intermolecular hydrogen bonding on the surface sugar chain of CHP NPs causes the rapid release of phase I, whereas the slow diffusion of drug entrapped in the hydrophobic core of the particle leads to the slow release of phase II.

We observed different loading and encapsulation efficiency at different feed ratios. The drug entrapment in the NP core increases, and the drug adsorption on the surface decreases because the rapid release of drug phase I is weakened and the phase II slow release is enhanced. This is shown with DZP-CHP NPs at feed ratio 1:5 in Figure 4, which is consistent with its results of the highest entrapment efficiency. The particle size of NPs also affects the drug release rate. In general, with NPs with the same kind of carrier material, the smaller the particle size, the larger the interfacial area, and the release rate is higher. DZPCHP $(1: 5)$ with the smallest particle size had the slowest release rate, whereas DZP-CHP $(1: 2)$ with the largest particle size had the fastest release rate. The possible reason is that DZP-CHP $(1: 5)$ with the strongest hydrophobic interaction, gathered closely, is in a more stable state, for slower internal drug release. The release rate of DZP-CHP $(1: 2)$ is the opposite, which is consistent with the previous results of particle size.
3.4. Thermodynamic Analysis of the Effect of DZP-CHP NPs on Polysorbate 80. ITC is often used to measure the connection properties of two substances. Because of the heat generated or absorbed during material binding, the change in the heat of the reaction system can be measured by titrating the polysorbate 80 into the solution of the DZPCHP NPs to reflect the conjugation of the two. In Figure $5,4 \mathrm{ml}$ of Tween 80 was added to produce a corresponding endothermic peak; with an increase in number of drops, the peak gradually decreased. With the 15th drop, the peak value reversed and became an exothermic peak pointed downwards. Mean \pm SD data are as follows: coverage, $2.70 \pm 0.372 ; K_{\mathrm{A}}\left(10^{5} / \mathrm{M}\right), 2.98 \pm 1.66 ; \triangle H(\mathrm{cal} / \mathrm{mol})$, $1710 \pm 311.4$; and $\triangle S(\mathrm{cal} / \mathrm{mol} / \mathrm{deg}), 30.8$ (Table 2). During the dropping of polysorbate 80 molecules into the CHP NP solution, polysorbate 80 is adsorbed onto the surface of the CHP NP. Figure 5 shows that the adsorption of polysorbate 80 to the surface of DZP-CHP NPs is an endothermic process [13], and the faint exothermic peak at the tail may be due to polysorbate 80 reacting with donepezil adhered to the NP surface or some of the drug being released and dissolved in polysorbate 80 [18]. According to the Gibbs free energy formula, the result is $\triangle G<0$, which indicates that the reaction is a spontaneous reaction. $K_{\mathrm{A}}\left(10^{5} / \mathrm{M}\right)$ was $2.98 \pm 1.66$, which indicates good affinity between the two. The coverage was $2.7 \pm 0.372$, so about 2.7 polysorbate 80 molecules could be adsorbed on the surface of one CHP. Since one CHP NP consisted of several CHPs, polysorbate 80 had better coverage on the surface of CHP NPs.

3.5. Characterization of CHP, DZP-CHP, and PS-DZP-CHP NPs. From TEM $(\times 30000)$ (Figure 6$)$, the prepared CHP, DZP-CHP, and PS-DZP-CHP NPs were uniform and spherical. From Table 3 and Figure 4, the mean \pm SD diameter of DZP-CHP NPs before and after drug loading was 257.5 \pm 3.05 and $266.3 \pm 4.46$, respectively, and the NP size was relatively uniform with mean \pm SD dispersion index 0.169 \pm 0.020 and $122 \pm 0.01$. The size of microspheres changed a little before and after drug loading, but the particle size was increased after emulsification, to a mean \pm SD of 335.2 \pm 5.46 . Positively or negatively charged NPs exhibit more drug release than do neutral NPs [30]. The mean zeta potential increased from $-2.81 \pm 0.27$ to $-0.66 \pm 0.04 \mathrm{mV}$; after that, CHP NPs encapsulate donepezil, which may be due to the molecular interaction between the polymer and donepezil. As compared with charged NPs, neutral NPs (zeta potential: $\pm 10 \mathrm{mV}$ ) phagocytosed by macrophages are significantly reduced in vivo [31,32], and phagocytosis is the main factor in removing NPs. Reducing phagocytosis may significantly extend the cycle time in the body [33]; therefore, DZP-CHP may have a longer cycle time in vivo than donepezil alone. Zeta potential decreased to $-2.22 \pm 0.86 \mathrm{mV}$ after polysorbate 80 was applied. The surface charge of bovine serum albumin is $-5.6 \mathrm{mV}$, according to Paillard et al., and the potential of NPs decreases after adsorption on the surface of NPs [34]. Therefore, in this study, the potential reduction of NPs in $0.7 \mathrm{mmol}$ polysorbate 80 emulsifier may also be related to the adsorption. Also, the increased particle size may lead to 

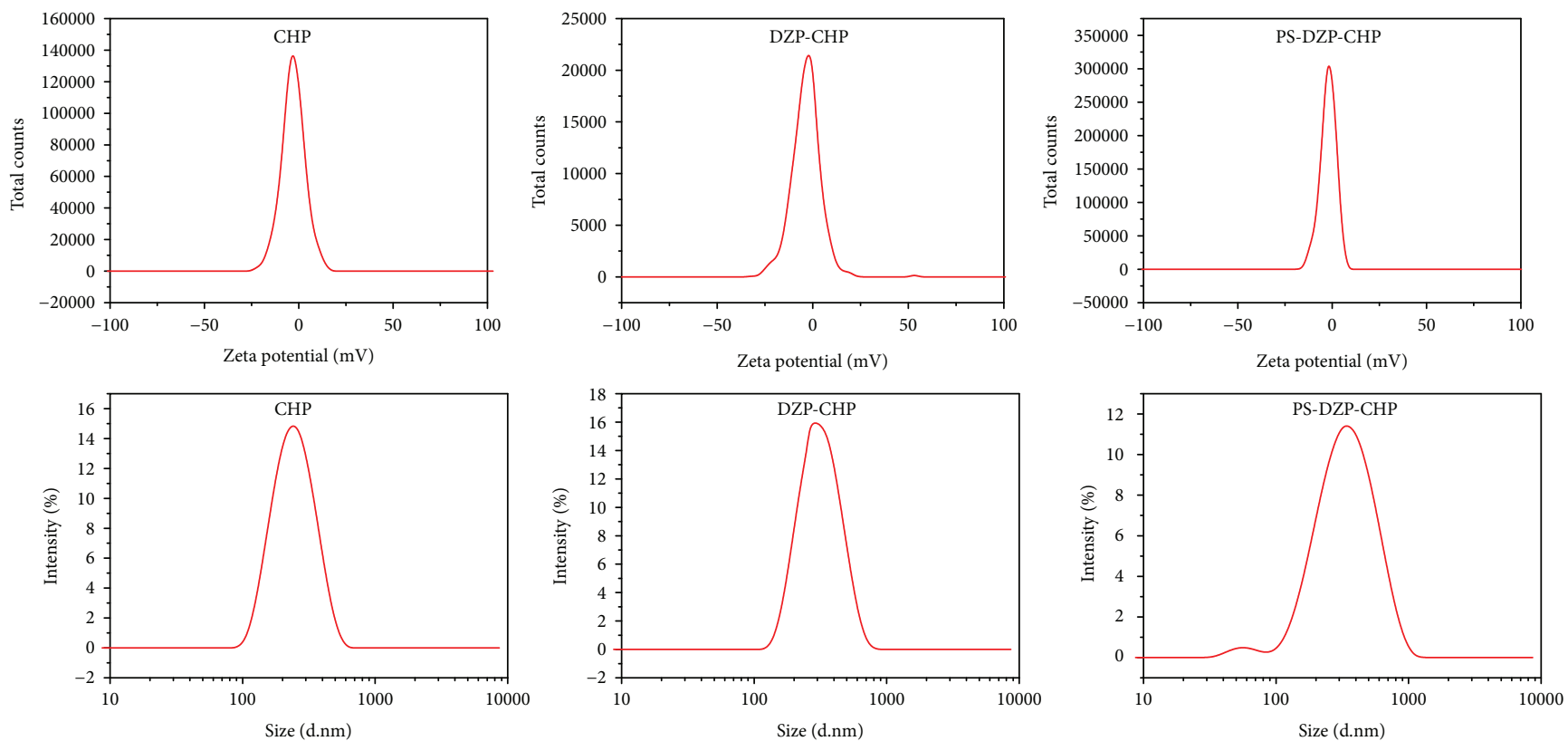

Figure 4: Particle size distribution and potential changes of NPs before and after drug loading. CHP: blank CHP NPs; DZP-CHP: donepezilloaded CHP NPs; PS-DZP-CHP: polysorbate 80-emulsified DZP-CHP.

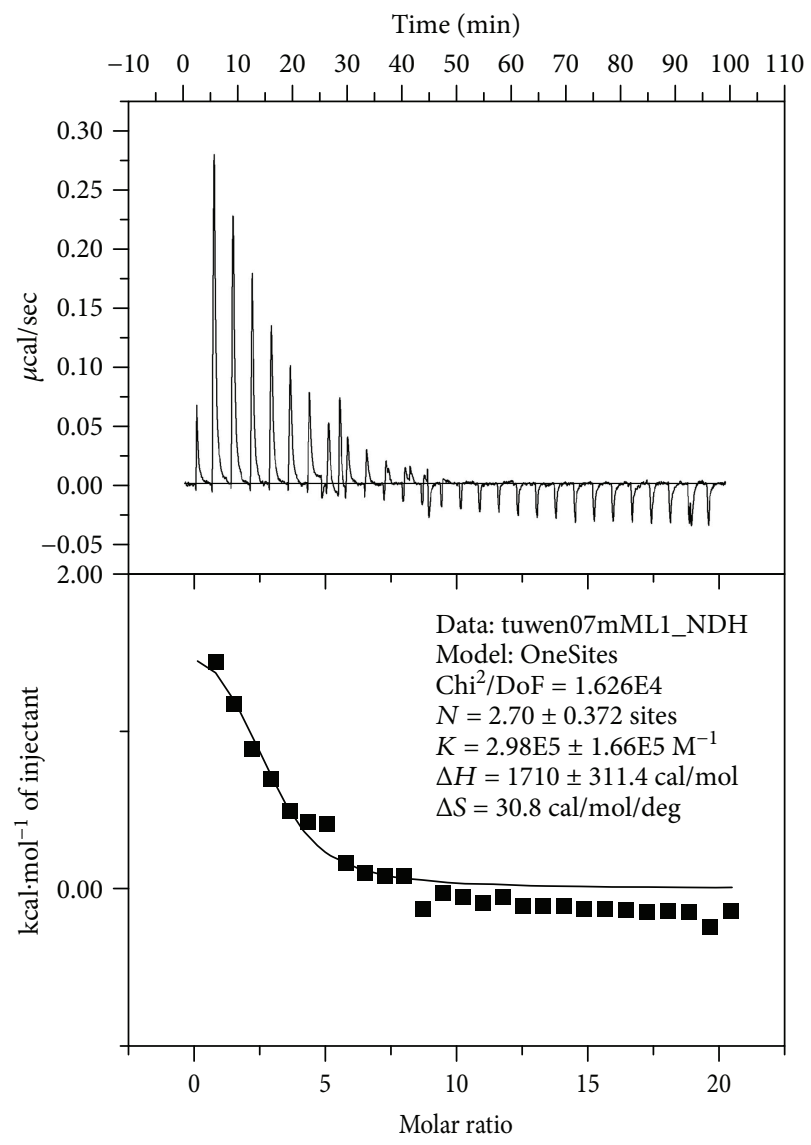

Figure 5: ITC data at $25^{\circ} \mathrm{C}$ for titration of polysorbate 80 into DZP-CHP NPs. The NP concentration in the cell $(250 \mu \mathrm{l})$ was $7.7 \mu \mathrm{M}$ and polysorbate 80 concentration in the syringe was $700 \mu \mathrm{M}$.
TABle 2: Affinities $\left(K_{\mathrm{A}}\right)$, degree of coverage, and enthalpy changes upon binding when polysorbate 80 was titrated into nanoparticle solution at $25^{\circ} \mathrm{C}$.

\begin{tabular}{lccc}
\hline Coverage & $K_{\mathrm{A}}\left(10^{5} / \mathrm{M}\right)$ & $\triangle H(\mathrm{cal} / \mathrm{mol})$ & $\triangle S(\mathrm{cal} / \mathrm{mol} / \mathrm{deg})$ \\
\hline $2.70 \pm 0.372$ & $2.98 \pm 1.66$ & $1710 \pm 311.4$ & 30.8 \\
\hline
\end{tabular}

reduced surface charge density of NPs, thereby decreasing its zeta potential value or because of the shielding effect of the NPs on the surface charge with the adsorbed polysorbate $80[1]$.

3.6. In Vitro Drug Release of DZP-CHP NPs Coated and Uncoated by Polysorbate 80. With donepezil alone (DZP), free drug release was complete within a very short time (Figure 7). With DZP-CHP NPs, the release was $55.12 \%$ in $72 \mathrm{~h}$, for a controlled release effect. With PS-DZP-CHP NPs, the release was $42.71 \%$ in $72 \mathrm{~h}$. With PS-DZP-CHP $\mathrm{NP}$ dialysis in fetal bovine serum (FBS), the drug release was $49.36 \%$ in $72 \mathrm{~h}$. With PS-DZP-CHP NPs in FBS, the drug release was $31.21 \%$ in $72 \mathrm{~h}$. When compared with the DZPCHP NPs, the drug release of PS-DZP-CHP NPs is slower. The reason for the slow release of the PS-DZP-CHP NPs may be the presence of polysorbate 80 around the NPs; the hydrophobic regions of polysorbate 80 have strong adsorption properties for hydrophobic small-molecule drugs, thereby hampering the release of the drug to the media. And when compared with the PS-DZP-CHP NPs, the drug release of PS-DZP-CHP NPs in FBS is slower. The reason for the slow release of PS-DZP-CHP NPs in FBS can be that NPs were always adsorbed to plasma proteins in the blood circulation possibly the ApoE. After adsorption of plasma proteins, drug release can be reduced due to the steric hindrance of proteins. The release of PS-DZP-CHP NPs 


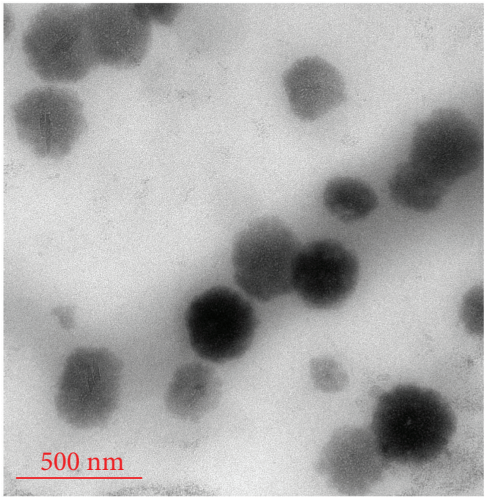

(a)

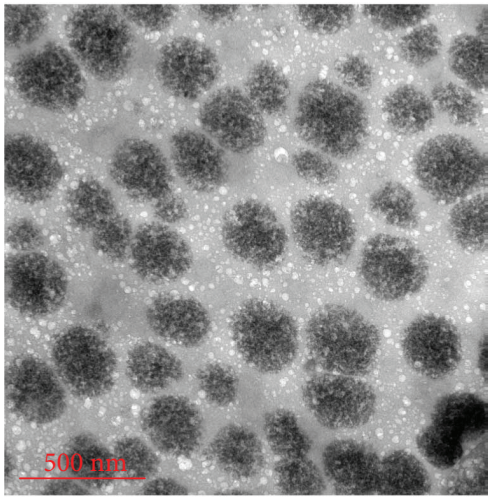

(b)

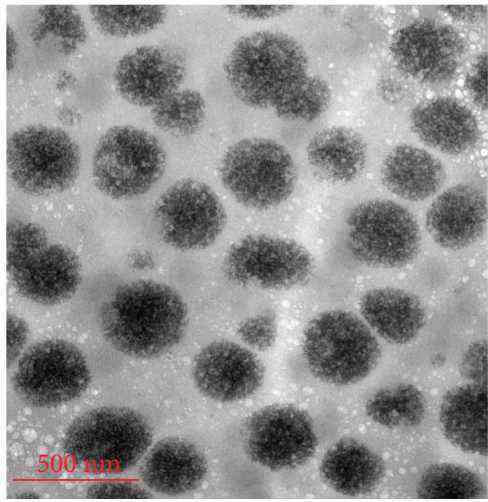

(c)

Figure 6: TEM of (a) CHP NPs, (b) DZP-CHP NPs, and (c) PS-DZP-CHP NPs.

TABle 3: Particle size, polydispersity index (PDI), zeta potential, drug loading, and entrapment efficiency of the three kinds of CHP NPs.

\begin{tabular}{lccccc}
\hline NPs & Particle size $(\mathrm{nm})$ & PDI & Zeta potential $(\mathrm{mV})$ & Drug loading (\%) & Entrapment efficiency (\%) \\
\hline CHP & $257.5 \pm 3.05$ & $0.169 \pm 0.02$ & $-2.81 \pm 0.27$ & - & - \\
DZP-CHP & $266.3 \pm 4.46$ & $0.122 \pm 0.01$ & $-0.66 \pm 0.04$ & $14.78 \pm 1.19$ & $88.77 \pm 2.60$ \\
PS-DZP-CHP & $335.2 \pm 5.46$ & $0.138 \pm 0.03$ & $-2.22 \pm 0.86$ & $8.25 \pm 1.80$ & $91.28 \pm 4.57$ \\
\hline
\end{tabular}

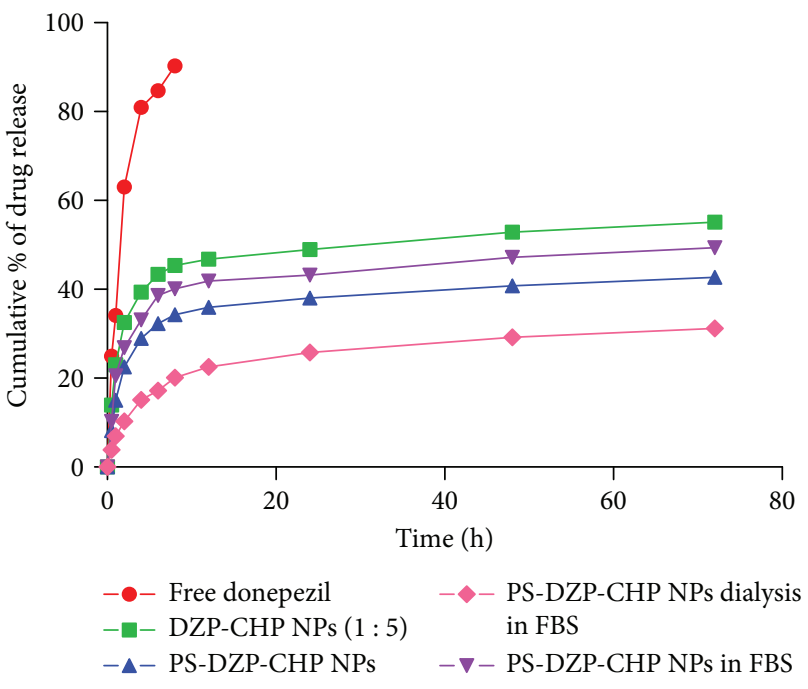

Figure 7: Release curves of donepezil from free donepezil, DZPCHP NPs (1:5), PS-DZP-CHP NPs, and PS-DZP-CHP NP dialysis in FBS and PS-DZP-CHP NPs in FBS.

dialysis in FBS is faster than PS-DZP-CHP NPs. The reason for the fast release of PS-DZP-CHP NPs dialysis in FBS may be that the drug adsorption of plasma proteins out of the Visking dialysis tubing and the concentration difference have been expanded.

\section{Discussion}

We found that pullulan can be modified into an amphiphilic polymer by bonding hydrophobic small-molecule groups, which can be used in the field of biomedicine. Akiyoshi et al., Deguchi et al., and Lee and Akiyoshi's studies [35-38] used hexamethylene diisocyanate as a linker and found that the hydrophobic cholesteryl molecular-modified pullulan could obtain the amphiphilic cholesteryl base chitosan (CHP), which can form self-assembled NPs in water. The NP hydrophobic center can load epirubicin, mitoxantrone, paclitaxel [39], and other fat-soluble drugs to enhance their solubility. However, the hexamethylene diisocyanate itself is flammable and belongs to the body's exogenous substances and may have toxic effects on humans. Succinic anhydride is easily degradable in the body of the endogenous substances in the TCA cycle. Therefore, we used succinic anhydride as the connecting arm to graft cholesteryl onto the CHP NPs (Figure 8(a)), which is safer in theory as a drug carrier. The CHP self-polymerization group is a circular or oval hydrogel NP formed by the noncovalent hydrophobic interaction between the cholesterol groups [40]. Therefore, the greater the degree of substitution of grafted cholesterol, the stronger the hydrophobic interaction and the more stable the structure. However, because of strong hydrophobic action, a degree of substitution $>6 \%$ is not conducive to self-aggregation.

We used thermodynamic analysis of the adsorption of polysorbate 80 and DZP-CHP NPs. In general, the binding reaction results from hydrogen bonding, electrostatic interaction, hydrophobic interaction, and van der Waals force. Many scholars believe that when $\Delta H>0$ and $\Delta S>0$, the hydrophobic interaction is dominant. With $\Delta H<0$ and $\Delta S<0$, the hydrogen bond and van der Waals force are the main driving forces. With $\Delta H<0$ and $\Delta S<0$, the electrostatic force is the main force [41]. Therefore, according to our ITC findings, the hydrophobic interaction plays an 


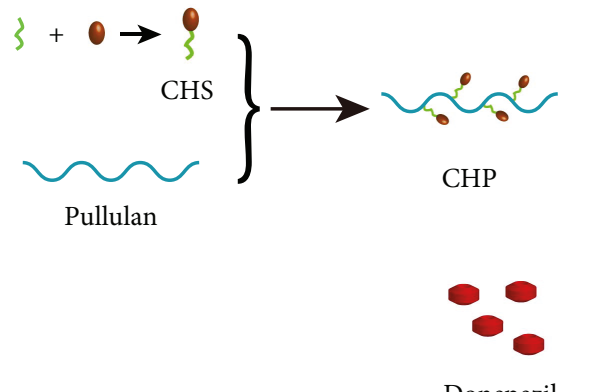

Donepezil

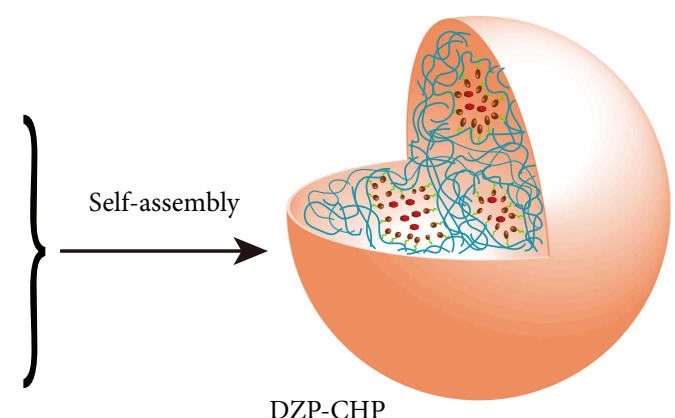

(a)

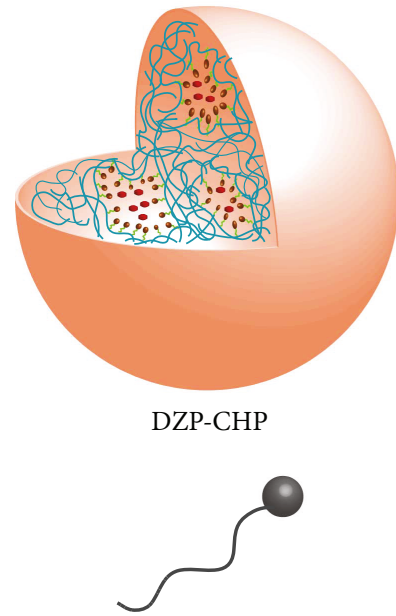

Polysorbate 80

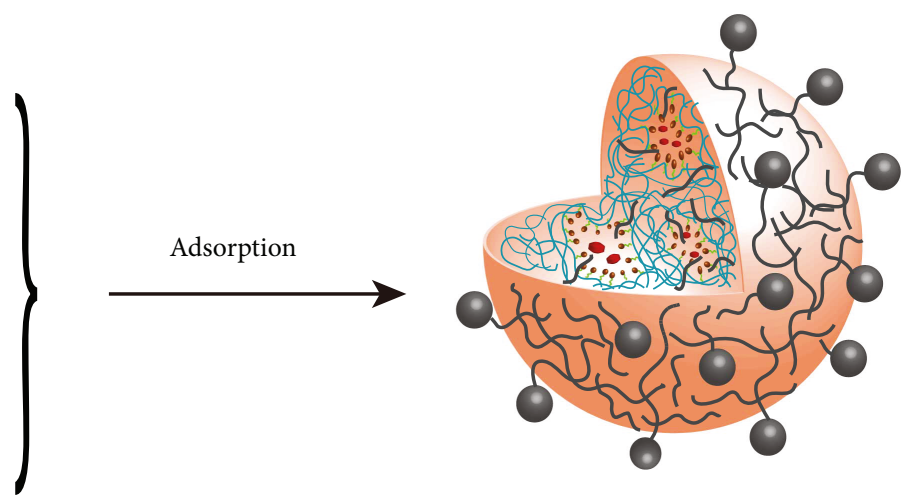

PS-DZP-CHP

(b)

Figure 8: (a) CHP synthesis and self-assembly with donepezil into drug-loaded NPs; (b) polysorbate 80 adsorbed on the drug-loaded CHP NPs surface.

important role in the interaction between DZP-CHP NPs and polysorbate 80 . According to Joshi et al. [42], polysorbate 80 may have structural changes in the initial phase of the adsorption of CHP NPs, and the acyl chain obtains a highly flexible structure. At the time of adsorption, the acyl chain $\left(-\mathrm{CH}_{2}\right)$ and ester group $(-\mathrm{C}=\mathrm{O})$ interacting with the CHP NPs under the hydrophobic interaction force dominate hydrogen bonding, so the polysorbate 80 molecule approaches the surface of the CHP NPs to result in the monolayer plane structure (Figure $8(\mathrm{~b})$ ), which is consistent with the experimental results.

Small particle size and other characteristics of the drug enhance its dissolution in the body and sustained release. In addition, studies have shown that NPs with polysorbate 80 coated on the surface are brain targeting and more permeable to the blood-brain barrier [43]. Polysorbate 80 is not cytotoxic and does not damage the blood-brain barrier of endothelial cells, making tight junctions appear to increase permeability for brain targeting [44]. The mechanism may be that polysorbate 80 coated on the surface of NPs can be used as an anchor point, and adsorption of apolipoproteins ApoB and ApoE occurs. The adsorption of apolipoprotein NPs mimics lipoprotein particles, which are absorbed by brain capillary endothelial cells via endocytosis mediated by the lipoprotein receptor low-density lipoprotein receptor and/or LRP [45-47]. In this case, the NPs traverse the blood-brain barrier as a Trojan horse bound to the drugs, and the drug is released into the endothelial cells, transported further into the brain by diffusion, or delivered to brain tissue by transcytosis to achieve brain targeting [48-50] or play the role of donepezil cholinesterase inhibitors to treat Alzheimer's disease [51, 52]. In addition, polysorbate 80 may also increase drug transport by hindering the specific exclusion of P-glycoprotein [53].

Polysorbate 80-coated NPs must be cross-linked to the blood-brain barrier with ApoB and ApoE proteins in the plasma. According to Ikai [54], polysorbate 80 is almost $1: 1$ bound to lipoprotein, and the binding is slow and tight. Both are in a stable state of reaction after $15 \mathrm{~h}$, which is advantageous for the slow release of the new dosage form in vivo for efficacy. Polysorbate 80 and lipoprotein have a cross-linking reaction; the string-like structure of varying length can be seen on electron microscopy (the structure is also likely between CHP cross-linked, inserted in the middle). At the same time, polysorbate 80 does not affect the physical structure of ApoE and low-density lipoprotein and is safer.

However, the adsorption of NPs and proteins is also affected by many factors, such as the hydrophobicity of NPs, zeta potential, surface curvature, and surface roughness. The former two factors are the most important. The stronger the hydrophobicity, the stronger the adsorption 
of protein and NPs. Since the material constituting the framework of the NP is a CHP molecule, the hydrophobic group is cholesteric and highly hydrophobic and easily interacts with the protein in the blood. Zeta potential also has an important effect on the adsorption of the two: in general, positively charged NPs preferentially adsorb proteins with isoelectric points $<5.5$, such as albumin, whereas negatively charged NPs are the opposite and the adsorption between the surface charge density increases. We found that the absolute value of the zeta potential was larger for the drug-loaded NPs coated with than without polysorbate 80 and negatively charged, so polysorbate 80 may enhance NP-protein adsorption, and this novel NP should bind preferentially to ApoE in the blood (isoelectric points of three Apo subtypes E2, E3, and E4 are $>5.5$ ) as compared with albumin.

We found that PS-DZP-CHP has small molecular hydrophobic drug solubilization and good sustainedrelease effect. The release experiment in vitro showed that the CHP NPs emulsified by polysorbate 80 can release drugs at about $42.71 \%$ in $72 \mathrm{~h}$, about $13 \%$ less than for nonemulsified NPs. In other words, the new NPs can be more stable in the blood, so they can maintain blood concentration longer, increase the medication interval, and improve patient compliance to treatment. Moreover, because of the increased stability, fewer drugs enter the systemic circulation and more NPs cross the blood-brain barrier before the release of the drug, thereby accumulating the drug in the brain and releasing it after targeting. The dosage can be reduced, as can the toxicity to the peripheral nervous system.

The controlled release of drug-loaded CHP NPs is an in vitro release behavior. When the NPs enter the body, the peripheral environment around the NPs is complicated and the release behavior is also complicated. However, due to the time and technical limitation, we did not confirm this theory by animal experiments, which needs further investigation.

\section{Conclusion}

The dose ratio of donepezil and nanomaterials has a certain effect on the drug loading, entrapment efficiency, and sustained-release features of CHP NPs, and the best feed ratio is 1:5. Surface modification of DZP-CHP NPs with polysorbate 80 showed good adsorption between polysorbate 80 and NPs on ITC. The CHP NPs have good drug loading, entrapment efficiency, and sustained-release effect, and the adsorption of polysorbate 80 further enhanced the sustained-release effect. CHP NPs after polysorbate 80 surface modification may be a new type of pharmaceutical preparation with a brain-targeting function in treating nervous system diseases.

\section{Conflicts of Interest}

The authors declare no conflict of interests regarding the publication of this paper.

\section{Authors' Contributions}

Xiaojun Tao and Yu Li contributed equally to this paper.

\section{Acknowledgments}

This research was supported by the Hunan Provincial Natural Science Foundation of China (no. 2017JJ2188), Advanced Fund of Hunan Provincial Education Department (no. 16A128), and Changsha Science and Technology Bureau Project (no. kq1701052) (Xing Feng).

\section{References}

[1] C. Riggio, E. Pagni, V. Raffa, and A. Cuschieri, "Nano-oncology: clinical application for cancer therapy and future perspectives," Journal of Nanomaterials, vol. 2011, Article ID 164506, 10 pages, 2011

[2] Y. Ito, M. Yoshimura, T. Tanaka, and K. Takada, "Effect of lipophilicity on the bioavailability of drugs after percutaneous administration by dissolving microneedles," Journal of Pharmaceutical Sciences, vol. 101, no. 3, pp. 1145-1156, 2012.

[3] H. Lu, J. Wang, T. Wang, J. Zhong, Y. Bao, and H. Hao, "Recent progress on nanostructures for drug delivery applications," Journal of Nanomaterials, vol. 2016, Article ID 5762431, 12 pages, 2016.

[4] S. M. Wong, I. W. Kellaway, and S. Murdan, "Fast-dissolving microparticles fail to show improved oral bioavailability," Journal of Pharmacy and Pharmacology, vol. 58, no. 10, pp. 1319-1326, 2006.

[5] Q. Lv, X. Li, B. Shen et al., "A solid phospholipid-bile saltsmixed micelles based on the fast dissolving oral films to improve the oral bioavailability of poorly water-soluble drugs," Journal of Nanoparticle Research, vol. 16, no. 6, p. 2455, 2014.

[6] S. I. Rapoport, "Osmotic opening of the blood-brain barrier: principles, mechanism, and therapeutic applications," Cellular and Molecular Neurobiology, vol. 20, no. 2, pp. 217-230, 2000.

[7] C. W. Tzeng, F. L. Yen, T. H. Wu et al., "Enhancement of dissolution and antioxidant activity of kaempferol using a nanoparticle engineering process," Journal of Agricultural and Food Chemistry, vol. 59, no. 9, pp. 5073-5080, 2011.

[8] C. W. Lee, F. L. Yen, H. W. Huang et al., "Resveratrol nanoparticle system improves dissolution properties and enhances the hepatoprotective effect of resveratrol through antioxidant and anti-inflammatory pathways," Journal of Agricultural and Food Chemistry, vol. 60, no. 18, pp. 4662-4671, 2012.

[9] A. Albanese, P. S. Tang, and W. C. W. Chan, "The effect of nanoparticle size, shape, and surface chemistry on biological systems," Annual Review of Biomedical Engineering, vol. 14, no. 1, pp. 1-16, 2012.

[10] W. Li, X. Zhao, X. Sun, Y. Zu, Y. Liu, and Y. Ge, "Evaluation of antioxidant ability in vitro and bioavailability of trans-cinnamic acid nanoparticle by liquid antisolvent precipitate," Journal of Nanomaterials, vol. 2016, Article ID 9518362, 11 pages, 2016.

[11] X. Jiang, Y. S. Cheng, and H. D. C. Smyth, "Nanostructured aerosol particles: fabrication, pulmonary drug delivery, and controlled release," Journal of Nanomaterials, vol. 2011, Article ID 198792, 2 pages, 2011. 
[12] R. Sakhtianchi, F. Atyabi, P. Yousef, E. Vasheghani-Farahani, M. Movahedi, and R. Dinarvand, "Targeted delivery of doxorubicin-utilizing chitosan nanoparticles surfacefunctionalized with anti-Her2 trastuzumab," International Journal of Nanomedicine, vol. 6, pp. 1977-1990, 2011.

[13] J. L. Molinuevo, M. L. Berthier, and L. Rami, "Donepezil provides greater benefits in mild compared to moderate Alzheimer's disease: implications for early diagnosis and treatment," Archives of Gerontology and Geriatrics, vol. 52, no. 1, pp. 18-22, 2011.

[14] I. Lynch, T. Cedervall, M. Lundqvist, C. Cabaleiro-Lago, S. Linse, and K. A. Dawson, "The nanoparticle-protein complex as a biological entity; a complex fluids and surface science challenge for the 21st century," Advances in Colloid and Interface Science, vol. 134-135, no. 21, pp. 167-174, 2007.

[15] P. Roach, D. Farrar, and C. C. Perry, "Surface tailoring for controlled protein adsorption: effect of topography at the nanometer scale and chemistry," Journal of the American Chemical Society, vol. 128, no. 12, pp. 3939-3945, 2006.

[16] X. Tao, Q. Zhang, K. Ling et al., "Effect of pullulan nanoparticle surface charges on HSA complexation and drug release behavior of HSA-bound nanoparticles," PLoS One, vol. 7, no. 11, article e49304, 2012.

[17] S. Boridy, H. Takahashi, K. Akiyoshi, and D. Maysinger, "The binding of pullulan modified cholesteryl nanogels to $\mathrm{A} \beta$ oligomers and their suppression of cytotoxicity," Biomaterials, vol. 30, no. 29, pp. 5583-5591, 2009.

[18] G. Kaur and S. K. Mehta, "Developments of polysorbate (Tween) based microemulsions: preclinical drug delivery, toxicity and antimicrobial applications," International Journal of Pharmaceutics, vol. 529, no. 1-2, pp. 134-160, 2017.

[19] R. N. Alyaudtin, A. Reichel, R. Löbenberg, P. Ramge, J. Kreuter, and D. J. Begley, "Interaction of poly(butylcyanoacrylate) nanoparticles with the blood-brain barrierin vivoandin vitro," Journal of Drug Targeting, vol. 9, no. 3, pp. 209-221, 2001.

[20] R. G. Strickley, "Solubilizing excipients in oral and injectable formulations," Pharmaceutical Research, vol. 21, no. 2, pp. 201-230, 2004.

[21] G. B. Patil and S. J. Surana, "Bio-fabrication and statistical optimization of polysorbate 80 coated chitosan nanoparticles of tapentadol hydrochloride for central antinociceptive effect: in vitro-in vivo studies," Artificial Cells, Nanomedicine, and Biotechnology, vol. 45, no. 3, pp. 505-514, 2017.

[22] Y. Wang, C. Wang, C. Gong et al., "Polysorbate 80 coated poly ( $\varepsilon$-caprolactone)-poly (ethylene glycol)-poly ( $\varepsilon$-caprolactone) micelles for paclitaxel delivery," International Journal of Pharmaceutics, vol. 434, no. 1-2, pp. 1-8, 2012.

[23] H. Azhari, M. Strauss, S. Hook, B. J. Boyd, and S. B. Rizwan, "Stabilising cubosomes with tween 80 as a step towards targeting lipid nanocarriers to the blood-brain barrier," European Journal of Pharmaceutics and Biopharmaceutics, vol. 104, pp. 148-155, 2016.

[24] N. Watrous-Peltier, J. Uhl, V. Steel, L. Brophy, and E. Merisko-Liversidge, "Direct suppression of phagocytosis by amphipathic polymeric surfactants," Pharmaceutical Research, vol. 9, no. 9, pp. 1177-1183, 1992.

[25] S. D. Tröster, U. Müller, and J. Kreuter, "Modification of the body distribution of poly(methyl methacrylate) nanoparticles in rats by coating with surfactants," International Journal of Pharmaceutics, vol. 61, no. 1-2, pp. 85-100, 1990.
[26] G. Pamunuwa, V. Karunaratne, and D. N. Karunaratne, "Effect of lipid composition on in vitro release and skin deposition of curcumin encapsulated liposomes," Journal of Nanomaterials, vol. 2016, no. 9, Article ID 4535790, 9 pages, 2016.

[27] X. Tao, Q. Zhang, W. Yang, and Q. Zhang, "The interaction between human serum albumin and cholesterol-modified pullulan nanoparticle," Current Nanoscience, vol. 8, no. 6, pp. 830-837, 2012.

[28] S. Kim, K. M. Park, J. Y. Ko et al., "Minimalism in fabrication of self-organized nanogels holding both anti-cancer drug and targeting moiety," Colloids and Surfaces B: Biointerfaces, vol. 63, no. 1, pp. 55-63, 2008.

[29] G. Baier, C. Costa, A. Zeller et al., "BSA adsorption on differently charged polystyrene nanoparticles using isothermal titration calorimetry and the influence on cellular uptake," Macromolecular Bioscience, vol. 11, no. 5, pp. 628-638, 2011.

[30] X. Tao, Y. Xie, X. Qiu, Q. Zhang et al., "Cholesterol-modified comparative study of amino-pullulan nanoparticles as a drug carrier, cholesterol-modified carboxyethyl pullulan and pullulan nanoparticles," Nanomaterials, vol. 6, no. 5, pp. 165-180, 2016.

[31] S.-D. Li and L. Huang, "Pharmacokinetics and biodistribution of nanoparticles," Molecular Pharmaceutics, vol. 5, no. 4, pp. 496-504, 2008.

[32] T. S. Levchenko, R. Rammohan, A. N. Lukyanov, K. R. Whiteman, and V. P. Torchilin, "Liposome clearance in mice: the effect of a separate and combined presence of surface charge and polymer coating," International Journal of Pharmaceutics, vol. 240, no. 1-2, pp. 95-102, 2002.

[33] D. P. Speert, F. Eftekhar, and Puterman M. L., "Nonopsonic phagocytosis of strains of Pseudomonas aeruginosa from cystic fibrosis patients," Infection and Immunity, vol. 43, no. 3, pp. 1006-1011, 1984.

[34] A. Paillard, C. Passirani, P. Saulnier et al., "Positively-charged, porous, polysaccharide nanoparticles loaded with anionic molecules behave as 'stealth' cationic nanocarriers," Pharmaceutical Research, vol. 27, no. 1, pp. 126-133, 2010.

[35] K. Akiyoshi, S. Deguchi, N. Moriguchi, S. Yamaguchi, and J. Sunamoto, "Self-aggregates of hydrophobized polysaccharides in water. Formation and characteristics of nanoparticles," Macromolecules, vol. 26, no. 12, pp. 3062-3068, 1993.

[36] K. Akiyoshi, S. Deguchi, H. Tajima, T. Nishikawa, and J. Sunamoto, "Microscopic structure and thermoresponsiveness of a hydrogel nanoparticle by self-assembly of a hydrophobized polysaccharide," Macromolecules, vol. 30, no. 4, pp. 857-861, 1997.

[37] S. Deguchi, K. Kuroda, K. Akiyoshi, B. Lindman, and J. Sunamoto, "Gelation of cholesterol-bearing pullulan by surfactant and its rheology," Colloids and Surfaces A: Physicochemical and Engineering Aspects, vol. 147, no. 1-2, pp. 203-211, 1999.

[38] I. Lee and K. Akiyoshi, "Single molecular mechanics of a cholesterol-bearing pullulan nanogel at the hydrophobic interfaces," Biomaterials, vol. 25, no. 15, pp. 2911-2918, 2004.

[39] Z. Liu, Y. Jiao, Y. Wang, C. Zhou, and Z. Zhang, "Polysaccharides-based nanoparticles as drug delivery systems," Advanced Drug Delivery Reviews, vol. 60, no. 15, pp. 1650-1662, 2008.

[40] K. Akiyoshi, T. Nishikawa, Y. Mitsui, T. Miyata, M. Kodama, and J. Sunamoto, "Self-assembly of polymer amphiphiles: thermodynamics of complexation between bovine serum 
albumin and self-aggregate of cholesterol-bearing pullulan," Colloids and Surfaces A: Physicochemical and Engineering Aspects, vol. 112, no. 2-3, pp. 91-95, 1996.

[41] P. D. Ross and S. Subramanian, "Thermodynamics of protein association reactions: forces contributing to stability," Biochemistry, vol. 20, no. 11, pp. 3096-3102, 1981.

[42] A. S. Joshi, A. Gahane, and A. K. Thakur, "Deciphering the mechanism and structural features of polysorbate 80 during adsorption on PLGA nanoparticles by attenuated total reflectance - Fourier transform infrared spectroscopy," RSC Advances, vol. 6, no. 110, pp. 108545-108557, 2016.

[43] Y. Chen and L. Liu, "Modern methods for delivery of drugs across the blood-brain barrier," Advanced Drug Delivery Reviews, vol. 64, no. 7, pp. 640-665, 2012.

[44] J. Kreuter, P. Ramge, V. Petrov et al., "Direct evidence that polysorbate-80-coated poly (butylcyanoacrylate) nanoparticles deliver drugs to the CNS via specific mechanisms requiring prior binding of drug to the nanoparticles," Pharmaceutical Research, vol. 20, no. 3, pp. 409-416, 2003.

[45] J. Kreuter, D. Shamenkov, V. Petrov et al., “Apolipoproteinmediated transport of nanoparticle-bound drugs across the blood-brain barrier," Journal of Drug Targeting, vol. 10, no. 4, pp. 317-325, 2002.

[46] J. Kreuter, T. Hekmatara, S. Dreis, T. Vogel, S. Gelperina, and K. Langer, "Covalent attachment of apolipoprotein A-I and apolipoprotein B-100 to albumin nanoparticles enables drug transport into the brain," Journal of Controlled Release, vol. 118, no. 1, pp. 54-58, 2007.

[47] P. Aggarwal, J. B. Hall, C. B. Mcleland, M. A. Dobrovolskaia, and S. E. Mcneil, "Nanoparticle interaction with plasma proteins as it relates to particle biodistribution, biocompatibility and therapeutic efficacy," Advanced Drug Delivery Reviews, vol. 61, no. 6, pp. 428-437, 2009.

[48] J. Kreuter, "Mechanism of polymeric nanoparticle-based drug transport across the blood-br,ain barrier (BBB)," Journal of Microencapsulation, vol. 30, no. 1, pp. 49-54, 2012.

[49] K. Prabhakar, S. M. Afzal, G. Surender, and V. Kishan, "Tween 80 containing lipid nanoemulsions for delivery of indinavir to brain," Acta Pharmaceutica Sinica B, vol. 3, no. 5, pp. 345-353, 2013.

[50] A. E. Gulyaev, S. E. Gelperina, I. N. Skidan, A. S. Antropov, G. Y. Kivman, and J. Kreuter, "Significant transport of doxorubicin into the brain with polysorbate 80 -coated nanoparticles," Pharmaceutical Research, vol. 16, no. 10, pp. 1564-1569, 1999.

[51] R. Stryjer, R. D. Strous, F. Bar et al., "Beneficial effect of donepezil augmentation for the management of comorbid schizophrenia and dementia," Clinical Neuropharmacology, vol. 26, no. 1, pp. 12-17, 2003.

[52] J. L. Cummings, P. A. Cyrus, F. Bieber et al., "Metrifonate treatment of the cognitive deficits of Alzheimer's disease," Neurology, vol. 50, no. 5, pp. 1214-1221, 1998.

[53] M. M. Nerurkar, P. S. Burton, and R. T. Borchardt, "The use of surfactants to enhance the permeability of peptides through Caco-2 cells by inhibition of an apically polarized efflux system," Pharmaceutical Research, vol. 13, no. 4, pp. 528-534, 1996.

[54] A. Ikai, "Extraction of the apo B cluster from human low density lipoprotein with tween 80," The Journal of Biochemistry, vol. 88, no. 5, pp. 1349-1357, 1980. 


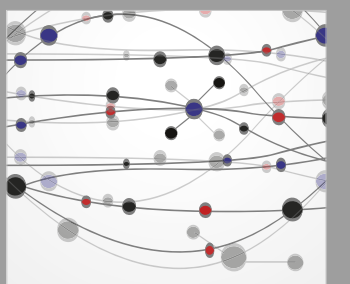

The Scientific World Journal
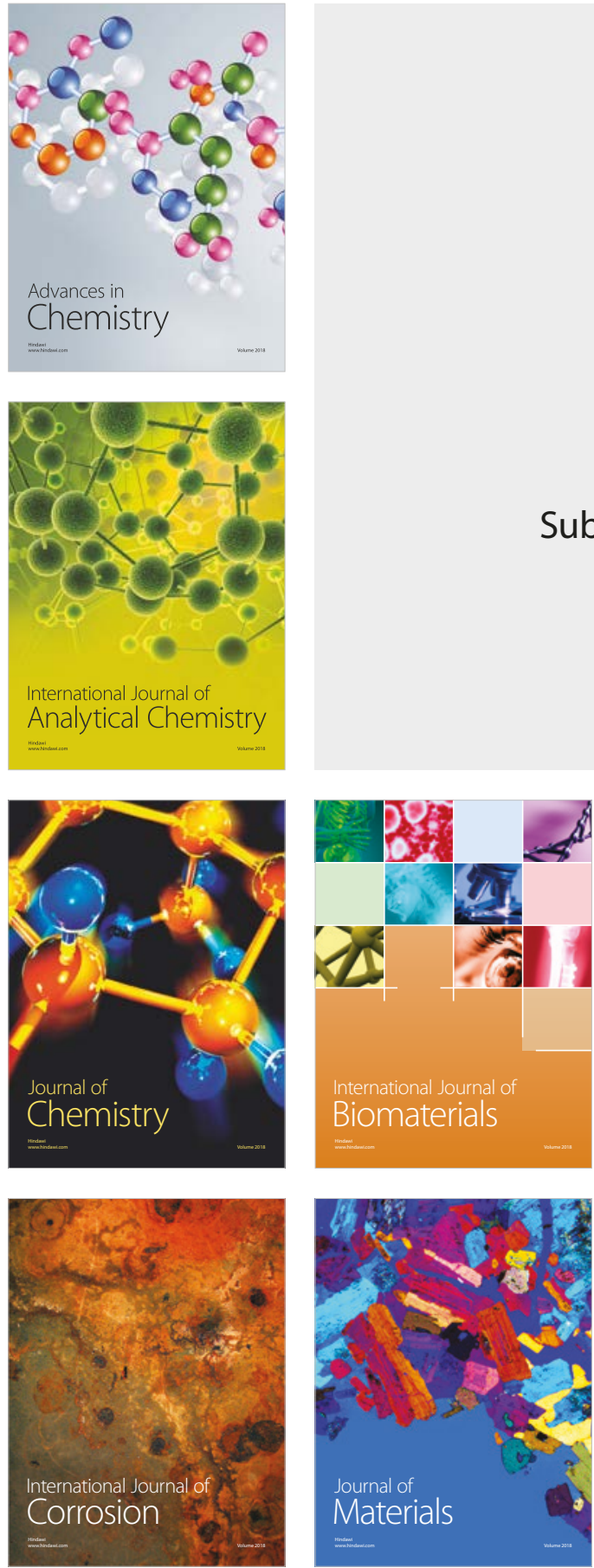

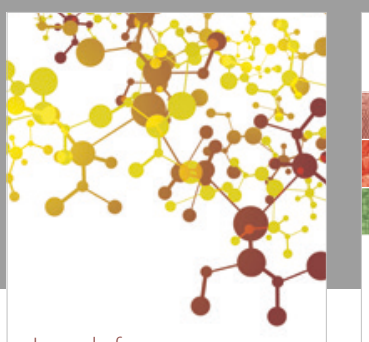

Journal of

Applied Chemistry
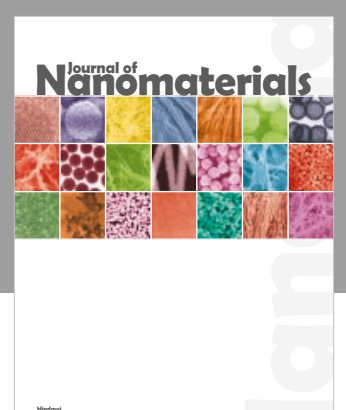

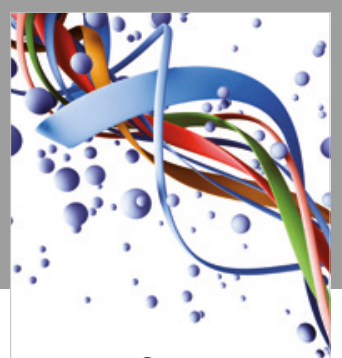

Scientifica

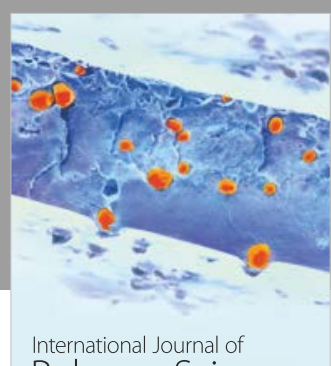

Polymer Science

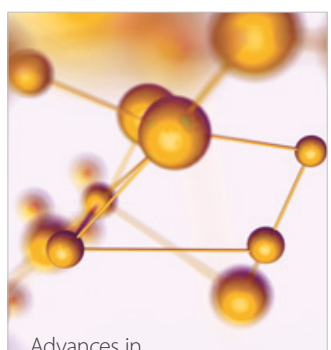

Physical Chemistry
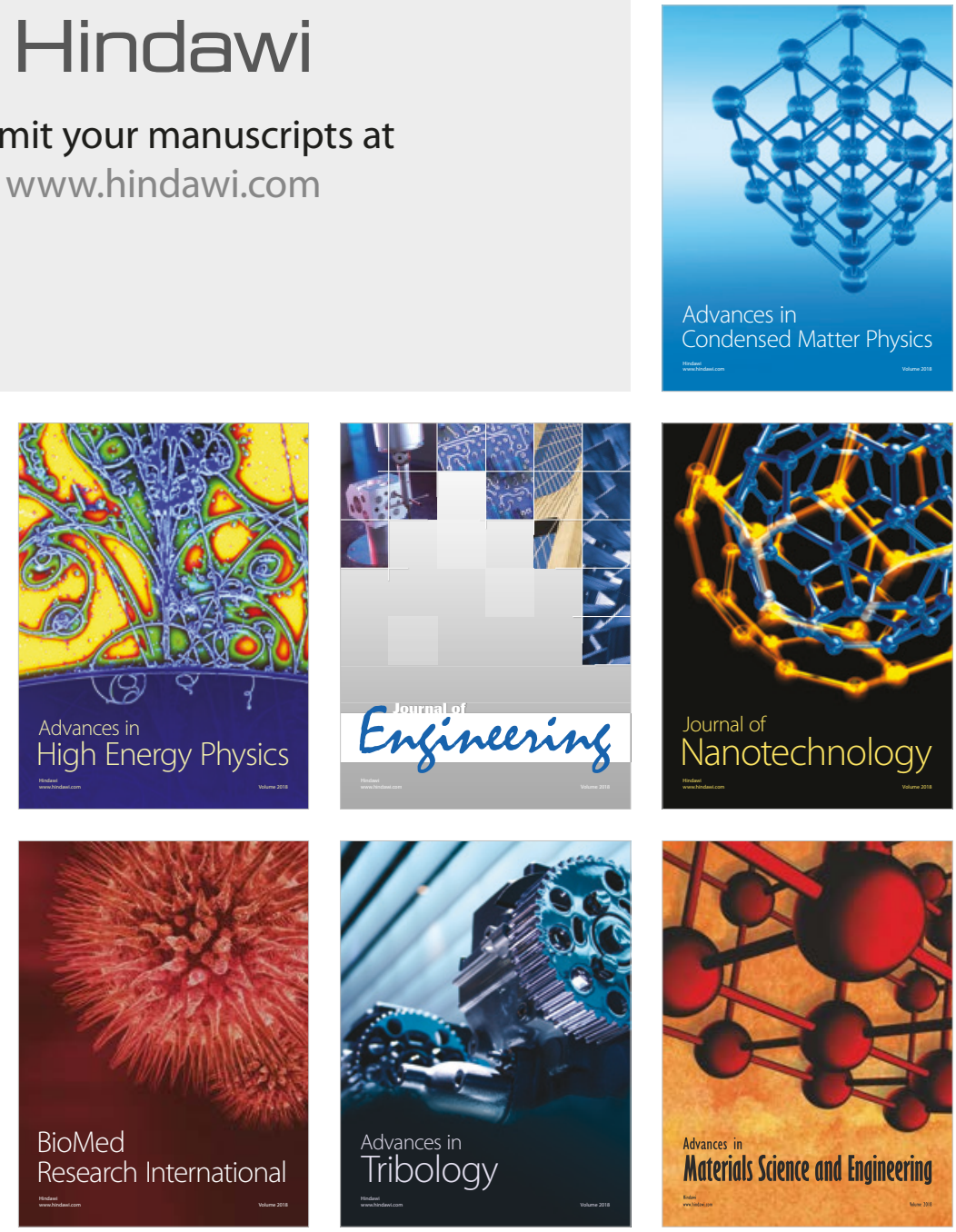